\title{
Relocation of facility location based on the inactive defense approach in humanitarian aid logis- tics
}

\author{
Reza Jalali ${ }^{*}$, Hossein Safari ${ }^{a}$, Mansour Momeni ${ }^{a}$ and Mohammadreza Sadeghi Moghadama
}

${ }^{a}$ Department of Industrial Management, University of Tehran, Tehran, Iran

\begin{tabular}{l}
\hline C H R O N I C L E \\
\hline Article history: \\
Received: November 26, 2017 \\
Received in revised format: Janu- \\
ary 31, 2018 \\
Accepted: April 7, 2018 \\
Available online: \\
April 8, 2018 \\
\hline Keywords: \\
Facility locating \\
Crisis \\
Humanitarian aid logistics
\end{tabular}

\section{Introduction}

In recent years, natural disasters and even man-made disasters have risen sharply. This has caused devastating effects and damages (Kunz \& Reiner, 2012; Broz et al., 2018). The increase in the incidences of catastrophes and the harmful and financial damages consequences will require a great deal of planning to deal with such incidents. Providing rescue and reconstruction of accident sites is one of the most important post-disaster actions that can restore hope and calm for the injured residents (Jafar Nejad et al., 2014). Operations and related actions aimed at preserving life and reducing people's suffering in crises are known as humanitarian operations. This operation involves the provision of materials and technical assistance, along with the provision of essential services under critical circumstances (Cozzolino, 2012). The ability of different communities to face these situations is different and is limited or reinforced by the resources, and planning of the countries. Humanitarian operations maintain a temporary nature and aim at re-establishing the self-sufficiency of affected communities (Sphere, 2004). Efforts to rescue and aid, at the time of the disaster, can reduce the damage and provide aid services to the survivors to reduce the suffering of the injured people (Esmaeili \& Barzinpour, 2014). The most important efforts at this time would be to transfer the injured to temporary hospitals and

\footnotetext{
* Corresponding author.

E-mail address: jalali.reza@live.com (R. Jalali)

(C) 2018 by the authors; licensee Growing Science, Canada doi: $10.5267 /$ j.msl.2018.4.022
} 
clinics, to evacuate affected people and to transfer them to safe havens. These efforts are heavily influenced by the location of facilities in aid logistics (Kawasaki et al., 2013).

Humanitarian logistics operations can be divided into two general categories of operations before and after the crisis. Pre-crisis operations include two types of activities. The first category is the activities that play the role of strategic planning. These activities include facility locating and inventory prepositioning. The second category is activities that play the role of crisis reduction. Defense and evacuation activities fall into this category. Post-crisis operations which start after the occurrence of the crisis, also include two categories of actions. The first category is activities that began shortly after the crisis and have a short-term nature, such as the distribution of aid supplies to the injured, the distribution of medical items to hospitals, the evacuation of refugees, the transfer and treatment of the injured. The second category is activities such as improving, rebuilding, and dealing with simultaneous crises, which are started a few days after the crisis and has a long-term nature (Cozzolino, 2012).

As noted, many aid actions are affected by locating the facilities. Therefore, this research also focuses on the locating of the facilities that play the role of strategic planning in pre-crisis activities. This also intends to provide suitable accommodation for facilities such as a hospital, outpatient clinics, police centers, distribution depots and temporary shelters, taking into account the principles of defense and using mathematical modeling.

\section{Theoretical Foundations}

\subsection{Humanitarian supply chain management}

Humanitarian aid, during and after the disasters, is aimed for preserving people's lives, alleviating human suffering and maintaining human dignity. Carrying out the humanitarian actions can enhance the aid power after a disaster. Meanwhile, what succeeds in humanitarian actions, is humanitarian supply chain management. The humanitarian chain management refers to planning, implementation and controlling the effectiveness of the flow and storage of goods and materials, as well as related information from point of origin to point of consumption, to reduce the suffering of the affected people. Various stages have been taken to address the humanitarian supply chain management, four main stages of human resource management which are in the most accepted view are defined as followings (Cozzolino, 2012; Van Wassenhove, 2006; Schulz \& Blecken, 2010; Oloruntoba \& Gray, 2006):

-Prevention: This stage includes rules and mechanisms that can reduce vulnerability. This usually involves the responsibility of governments.

- Preparation: refers to a variety of operations that take place before a disaster occurs. This stage involves steps that will make successful operations in the response. Facility locating is one of the important issues to be addressed at this stage.

-Response: A set of actions that start immediately after the crisis. At this stage, in collaboration with all actors, it is seeking immediate response to the damage caused.

-Reconstruction: This phase includes post-incident operations and includes rehabilitation and aims to address long-term problems.

\subsection{Facility locating}

Locating is a systematic activity, which is based on appropriate indicators and the best choice for a center, unit or service is suggested (Hooshiyar, 2010; Nidhi \& Pillai, 2017). Locating studies have been considered as one of the key elements in the success and survival of industrial, public and service centers (Hooshiyar, 2010), which can have economic effects on the performance of an industrial or 
service unit; social, environmental, cultural and economic impacts on the surrounding environment (Hall, 2012). In the humanitarian supply chain facilities locating models, the goal is to locate shelters, tents, distribution centers and aid supplies. Locational models can be used in conjunction with evacuation or pre-positioning locations.

\subsection{Passive defense}

Along with the increase in population and the creation of urban organizations, the issue of safety and defense have become more important. According to Jalali Farahani, (2011), the issue of defending has become more important in most cities, with more residents and the creation of urban organizations, more invasion has occurred, which has led to the deaths of millions. These cases define necessity to stringent principles and rules to make cities more secure against invasions. One of these principles is passive defense, which refers to all actions and measures that reduce the vulnerability of casualties and increase sustainability without using weapons. High concentration of population in cities increases the risk of urban dangers and crises. In this regard, passive defense is trying to provide the proper pattern of land allocation to the needs of the cities and their proper location in the city body in order to ensure the well-being and comfort of townspeople and the safety of the cities. Passive defense emphasizes that locating the facilities can minimize future risks. Based on this, it suggests that locating should be based on three principles of safety, adaptability and efficiency:

- Principle of safety: Based on this principle, the facility location should be safe from the risk of a crisis. For example, for safety reasons, the hospital's location should be safe and no dangerous should exist for the hospitals. Establishing a hospital can keep this distance away from hazardous centers such as industrial centers and not-confining (Hosseini et al., 2013).

- Compatibility principle: Compatibility means consistency, coordination and non-disturbance between two types of urban users. Users that fall into the sphere of influence must be in harmony with each other in terms of the similarity of activity, and they do not interfere with other activities (Ghaffari, 1998).

- Performance principle: The functionality of a user, it means that it's the right user. One of the important factors that increase the efficiency of a hospital or relief center during a crisis is to provide service to the injured in the shortest possible time (Hosseini et al., 2013).

This research seeks to apply the principles of passive defense in locating facilities in the humanitarian supply chain. In fact, this article seeks to find the right place for the hospital and outpatient clinics to have the most effective and maximum safety in the crisis and to be compatible with the rest of the user.

\section{Literature Review}

Facility locating models in the humanitarian supply chain are commonly considered simultaneously with pre-positioning inventory models and discharging or distributing the aid. The only absolute location models that decide on the construction or selection of facilities is provided by Dessouky et al. (2006) and Jia et al. (2007). These models consider the maximum coverage location with quantitative and quality limitations of coverage. Chen et al. (2006) presented an uncertainty model to minimize dissatisfaction for locating a strategic facility. Jia et al. (2007) proposed a unidirectional facility for locating uncertain facilities that would select the appropriate location for the location of emergency service facilities such as fire stations or sources including ambulances in an emergency. Balcik and Beamon (2008) presented a predetermined aid product locating model to determine the number, location and capacity of distribution centers in sudden disasters. In this model, they considered budget constraints before and after the crisis.

Rawls and Turnquist (2011) presented a mixed planning model of a two-stage probabilistic integer, in which the model is decided on the location of the facility and the level of inventory for aid supplies. In 
this model, the amount of demand, the remaining amount of available inventory and the position of the transportation network are determined in the form of uncertainty. Jones (2011) focused on locating facilities before the disaster. He has provided the right place for facilities, taking into account the facility distance to the points of damage and the possibility of a failure of the facility. Naji-Azimi et al. (2012) presented a locating model in which appropriate distribution centers were selected from a number of potential points and people came to these places to receive aid items. The need for these distribution centers has also been resolved by a supplier. To solve this model, an innovative local search algorithm has been modified. Rawls and Turnquist (2012) presented a dynamic locational model for responding to short-term demand under uncertainty. In this model, the goal is to increase the satisfactory demand, taking into account reliability. Akgün et al. (2014) looked to find the appropriate location for facilities in such a way that the risk of non-coverage of demand is minimized. Zhang et al. (2006) proposed a reliable location-based model of risk taking into account the disturbance in the chain. In their research, the probability of failure of the facility is considered, and in the event of a malfunctioning of the facility assigned to one point at a level, another facilitator will be allocated at the next level, and if all facilities allocated to a point of injury, the fee is considered to be fine. Campbell and Jones et al. (2011) presented a multi-objective programming model for locating and assigning three-level fuzzy states and then solving them using a genetic algorithm.

\section{Modelling}

As mentioned, in this research, the goal is to determine the appropriate location for facilities, taking into account the passive defense principles. In fact, suitable locations for aid hospitals and outpatient clinics are selected based on the following indicators:

Population density: shows the average population of a region (region, sector) to the total area of the region (region, sector), which plays an important role in determining the service providers. Accordingly, in urban areas with high population density, it is more likely to be vulnerable than other areas (Hosseini et al., 2013). Therefore, in this research, the priority of creating health centers is close to those areas that are more densely populated. Applicability: Use of health centers (hospitals and outpatient clinics) should not be in conflict with the other uses. In fact, health centers should be built where the use of it is not in conflict with the use of area Accordingly, the matrix of compatibility of the user in terms of compatibility can be: a) completely incompatible; b) relatively compatible; c) indifferent; d) compatible; e) is fully compatible. After identifying different uses of different regions of Boushehr, the extent of user proportionality of different regions with the user to create the treatment areas is as follows.

Table 1

The extent of user proportionality of different regions with the user

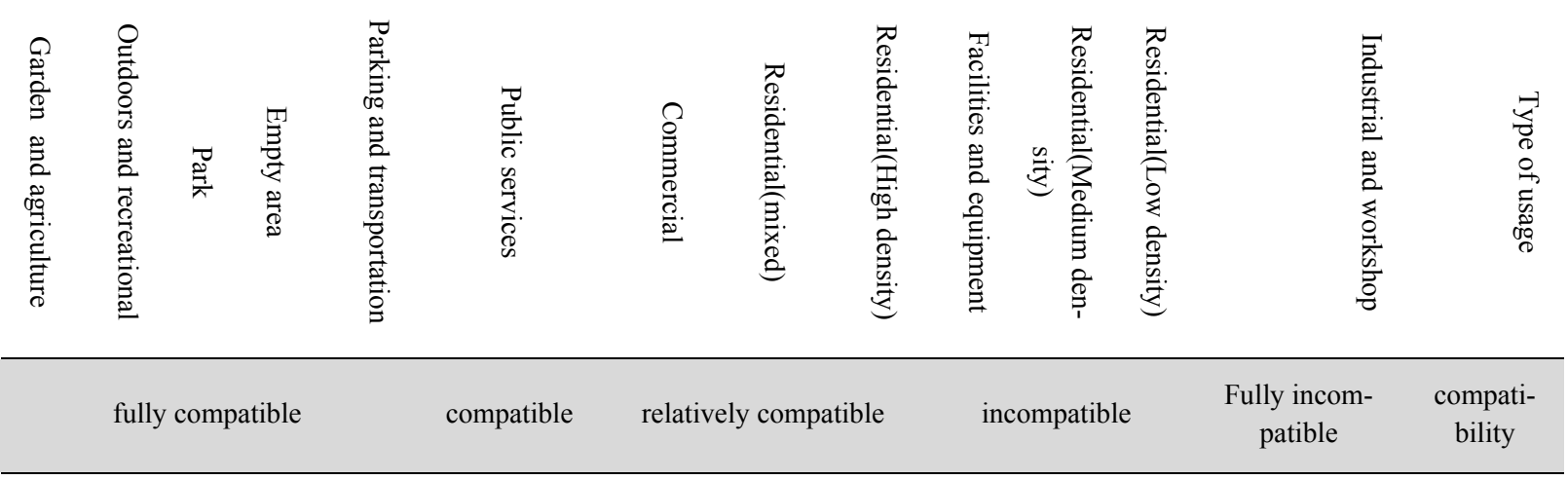


Then, for each urban area, the user-friendliness index for that area is calculated for the area with the above-mentioned compatibility. Access to the way: Health centers should be built in a place that has access to the way. If the health centers are built in areas where the hierarchy of communication networks is not met and the width of the roads in that area is low, a high severity of the crisis will result in the loss of the efficiency of the communication networks. In this research, we have used the width of the road to express road access. Accordingly, for each area, the access ways to the road is calculated in terms of access to more widespread roads. Enclosure: One of the important issues that can help to relieve the injured is the blocking of roads. At the time of the crisis, the need to evacuate the injured and to provide aid in the shortest possible time is presented, and this is most often done through intercity roads, interstate streets and suburbs. If one of these passes closes, the aid time will multiply. In the case of this index, we assume that low-lying buildings with a higher tread width (lower degree of enclosure) are more maneuverable, since the volume of rubble in those tracks is less. Also, buildings with higher altitudes are more likely to be degraded and more vulnerable. Accordingly, the degree of enclosure of each damage point is calculated based on the number of floor of buildings in that area. After describing the key indicators for locating the health facility, the dimensions of the model are described:

\section{Assumptions}

1. The earthquake does not occur in discrete areas/locations, but in this research, each earthquake area has a section or cluster of earthquake that includes a large number of houses and buildings that are affected. But instead of considering each of them as an earthquake area, that complicates the problem, we divide it into several sections.

2. The degree of population density, user appropriateness, degree of confinement, level of access, and the degree of neighborhood of each area can be measured and varied.

3. Hospital centers and aid stations are located in damaged areas in such a way that they can directly serve some of the injuries that are predetermined.

4. The centers of the main warehouses directly supply the goods and materials to the affected people, where the places are not predefined.

5. Shelters are safe areas where some of the injured are transferred to it, and its location is not preset.

6. The distance between the facilities is clear.

\section{The model indices}

Set of the damage points $(i \in I)$

Set of the warehouse $(w \in W)$

Set of the outpatient clinics $(k \in K)$

Set of the hospitals $(h \in H)$

Set of the shelters $(s \in S)$

\section{The model parameters:}

$d e_{i}::$ The amount of demand for damaged points I

$d_{i k}:$ The distance between the injury point $\mathrm{i}$ and outpatient clinic $\mathrm{k}$

$d_{i h}$ : Distance between injury point $\mathrm{i}$ and hospital $\mathrm{h}$

$d_{k h}$ : Distance between outpatient clinic $\mathrm{k}$ and hospital $\mathrm{h}$

$d_{i s}$ : Distance between damage point $\mathrm{i}$ and shelter $\mathrm{s}$

$d_{w s}$ : Distance between warehouse w and shelter $\mathrm{s}$

$d_{w k}$ : Distance between warehouse $\mathrm{w}$ and outpatient clinic $\mathrm{k}$ 
$d_{w h}:$ Distance between warehouse $\mathrm{w}$ and hospital $\mathrm{h}$

$\operatorname{cap}_{k}$ : Outpatient Clinic Capacity

$\operatorname{cap}_{h}:$ Hospital Capacity h

cap $_{s}:$ Shelter capacity s

$\operatorname{cap}_{w}$ : Warehouse capacity $\mathrm{W}$

$t a_{i}$ : Population density at injury points i

$t o_{i}$ : user appropriateness at points of injury i

$d m_{i}$ : Level of access Points of damage i

$t o_{i}$ : Enclosure of damage points $\mathrm{i}$

$\mathrm{C}$ : Total available budget

P: Number of outpatient outpatient clinics to be built.

q: Number of shelters to be constructed.

A: The number of warehouses to be built.

Q: Number of hospital to be constructed.

$\beta_{i}$ : The probability of injury and the need for treatment at injury points $\mathrm{i}$

r: Required items for healthy people in shelter

f: Required items for injured people

\section{The decision variables:}

$y_{s}$ : If $\mathrm{s}$ is chosen as a haven, then it is equal to one, otherwise is equal to zero.

$e_{h}:$ If $\mathrm{h}$ is chosen as a hospital, then one is equal to one, otherwise is equal to zero.

$u_{w}:$ If $\mathrm{w}$ is chosen as the warehouse, it is equal to one, otherwise is equal to zero.

$z_{k}$ : If $\mathrm{k}$ is chosen as an outpatient treatment center, then it is equal to one, otherwise is equal to zero

$\psi_{i k}$ : Number of people transferred from the injury point I to the outpatient clinic $\mathrm{k}$

$\delta_{i h}$ Number of people transferred from the injury point I to the hospital h

$\varphi_{i s}$ : The number of people transferred from the injury point $\mathrm{i}$ to the shelter $\mathrm{s}$

$\gamma_{i h}$ : Number of people transferred from outpatient clinic $\mathrm{k}$ to hospital $\mathrm{h}$

$\theta_{w s}$ : The amount of items transferred from the warehouse $\mathrm{w}$ to the shelter $\mathrm{s}$

$\tau_{w h}:$ The amount of items transferred from warehouse $\mathrm{w}$ to the hospital $\mathrm{h}$

$\phi_{w k}:$ The amount of items transferred from warehouse $\mathrm{w}$ to outpatient clinic $\mathrm{k}$

$I_{k}:$ Number of people kept in outpatient clinics $\mathrm{k}$

The designed model has three types of minimization. The first objective function states that hospital centers should be located in areas that are close to those areas where user appropriateness, population density, and access are more distant, and are more distant from areas of greater confinement (function 1). Outpatient centers are also selected on this basis (function 2). Temporary shelters should also be constructed at points where the total distance from damage points is minimized. In addition, warehouses should also be constructed in places where the total distance from shelters, hospitals and outpatient clinics can be minimized (function 3). The fourth limitation is the existence of balance flow in the outpatient treatment centers. Fifth limitation represents the percentage of population in each region that should be treated in a hospital or outpatient treatment center. The sixth limit represents the percentage 
of the population of each region that must be transferred to the shelter and do not need treatment. The seventh limitation represents the maximum capacity of any outpatient treatment. The eighth limitation represents the maximum capacity of each shelter. The minimum requirements for each shelter, the maximum capacity of the warehouses and the volume of the flow of items required from shelves to the shelter are expressed by the 9th to 11 th limits. The maximum capacity of each hospital for accepting injured people and items required from warehouses to outpatient clinics and hospitals is specified by the 12th and 13th limits. The injured person can be transferred from the health center $\mathrm{k}$ to the hospital $\mathrm{h}$, which was established by the hospital (limitation 14). The wounded person is transmitted from the injury point to the health center $\mathrm{k}$ where that area is established (limitation 15). The wounded person is transmitted from the injury point to the hospital $\mathrm{h}$, when the hospital was established (limitation 16). Persons will be transferred from the point of injury to the Shelter S when that shelter has been established (limitation 17). The goods are transferred from the warehouse w to the shelter s when that shelter was created (limitation 18). The goods will be transferred from the warehouse w to the hospital $h$ when that hospital is established (limitation 19). The goods are transferred from the storehouse w to the health center $\mathrm{k}$ when the treatment center is established (limitation 20). Limits 21 to 24 indicate the number of relief centers, hospitals, warehouses and shelters to be constructed, respectively.

$$
\begin{aligned}
& \min \mathrm{z}_{1}=\sum_{i} \sum_{h}\left(t a_{i} d_{i h}\right) \mathrm{e}_{h}+\sum_{i} \sum_{h}\left(t o_{i} d_{i h}\right) \mathrm{e}_{h}+\sum_{i} \sum_{h}\left(d m_{i} d_{i h}\right) \mathrm{e}_{h}-\sum_{i} \sum_{h}\left(m a_{i} d_{i h}\right) \mathrm{e}_{h} \\
& \min \mathrm{z}_{2}=\sum_{i} \sum_{k}\left(t a_{i} d_{i k}\right) \mathrm{z}_{k}+\sum_{i} \sum_{k}\left(t o_{i} d_{i k}\right) \mathrm{z}_{k}+\sum_{i} \sum_{k}\left(d m_{i} d_{i k}\right) \mathrm{z}_{k}-\sum_{i} \sum_{k}\left(m a_{i} d_{i k}\right) \mathrm{z}_{k} \\
& \min \mathrm{z}_{3}=\sum_{i} \sum_{s} d_{i s} y_{s}+\sum_{w} \sum_{s} d_{w s} \mu_{w}+\sum_{w} \sum_{h} d_{w h} u_{w}+\sum_{w} \sum_{k} d_{w k} u_{w}
\end{aligned}
$$

subject to

$$
\begin{aligned}
& \sum_{i} \psi_{i k}=\sum_{h} \gamma_{k h}+I_{k} \\
& \sum_{k} \psi_{i k}+\sum_{h} \delta_{i h}=\beta_{i}+\mathrm{de}_{i} \\
& \sum_{s} \varphi_{i s}=\left(1-\beta_{i}\right)+\mathrm{de}_{i} \\
& \sum_{k} \psi_{i k} \leq c a p_{k} \mathrm{z}_{k} \\
& \sum_{k} \varphi_{i s}+\sum_{k} \theta_{w s} \leq \mathrm{cap}_{s} \mathrm{y}_{s} \\
& \sum_{w} \theta_{w s} \leq r \sum_{i} \varphi_{i s} \\
& \sum_{k} \theta_{w s}+\sum_{w} \tau_{w h}+\sum_{w} \phi_{w k} \leq \mathrm{cap}_{w} \\
& \sum_{w} \sum_{s} \theta_{w s} \geq \mathrm{r} \sum_{i} \sum_{s} \varphi_{i s} \\
& \sum_{i} \delta_{i h}+\sum_{j} \gamma_{k h} \leq \operatorname{cap}_{h}
\end{aligned}
$$


$\sum_{w} \sum_{j} \phi_{w j}+\sum_{w} \sum_{h} \tau_{w h} \geq \mathrm{f}(1-\beta) \mathrm{de}_{i}$

$\sum_{k} \gamma_{k h} \leq M \mathrm{e}_{h}$

$\sum_{i} \psi_{i k} \leq M \mathrm{z}_{k}$

$\sum_{i} \delta_{i h} \leq M \mathrm{e}_{h}$

$\sum_{i} \varphi_{i s} \leq M y$

$\sum_{w} \theta_{w s} \leq M y_{s}$

$\sum_{w} \tau_{w h} \leq M \mathrm{e}_{h}$

$\sum_{w} \phi_{w k} \leq M \mathrm{z}_{k}$

$\sum_{k} \mathrm{z}_{k}=P$

$\sum_{h} e_{h}=Q$

$\sum_{w} u_{w}=A$

$\sum_{s} y_{s}=q$

$y_{s}, e_{h}, u_{w}, z_{k} \in\{0,1\} \quad, \psi_{i k}, \delta_{i h}, \varphi_{i s}, \gamma_{k h}, \theta_{w s}, \tau_{w h}, \phi_{w k}, I_{k} \in$ Integer $^{+}$

\section{Results}

To solve the above model, a lexicographic method has been used. In this method, each objective is firstly determined in order of importance in decision making, and then the optimal answer for several single-objective problems is calculated sequentially, in which the optimal solution of the $i^{\text {th }}$ single objective problem is entered in the $(i+1)^{\text {th }}$ problem solving space as a constraint. Consider the following multi-purpose problem:

$\min \mathrm{f}_{1}(x), \mathrm{f}_{2}(x), \ldots, \mathrm{f}_{k}(x)$

subject to

$\mathrm{g}_{i}(x) \leq 0, \mathrm{i}=1,2, \ldots, \mathrm{m}$

To solve this problem, the following steps are done by the lexicography method: 
$\min \mathrm{f}_{1}(x)$

subject to

$\mathrm{g}_{i}(x) \leq 0, \mathrm{i}=1,2, \ldots, \mathrm{m}$

After solving the problem, its solutions are obtained in the form, $x_{1}^{*}$ and $\mathrm{f}_{1}\left(x^{*}\right)=z_{1}{ }^{*}$, then the second problem is formulated as follows:

$\min \mathrm{f}_{2}(x)$

subject to

$\mathrm{g}_{i}(x) \leq 0, \mathrm{i}=1,2, \ldots, \mathrm{m}$

$\mathrm{f}_{1}\left(x^{*}\right)=z_{1}^{*}$

The answer of this problem will also be obtained in the form $x_{2}{ }^{*}$ and $\mathrm{f}_{2}\left(x^{*}\right)=z_{2}{ }^{*}$. This method continues until all the $\mathrm{k}$ numbers of the objective functions are investigated. The $J$ th model of the problem will be as follows:

$\min \mathrm{f}_{j}(x)$

subject to

$\mathrm{g}_{i}(x) \leq 0, \mathrm{i}=1,2, \ldots, \mathrm{m}$

$\mathrm{f}_{t}\left(x^{*}\right)=z_{t}^{*}, \mathrm{t}=1, . ., \mathrm{j}-1$

The answer of this problem will also be obtained in the form $x_{j}^{*}$ and $\mathrm{f}_{j}\left(x^{*}\right)=z_{j}{ }^{*}$. Finally, the answer is named $x_{k}{ }^{*}$ and considered as the optimal answer of the main multipurpose optimization problem. In this research, considering the first to third priority for the first, second and third objective functions, and using the lexicography method, the answers of the problem are obtained.

The case study of this research is port of Boushehr, which is divided into 30 regions. Accordingly, using the GIS software, the parameters of distance between points, and population density, user appropriateness, level of access, and confinement for each area are estimated. Then, according to experts' idea, 12, 10, 10, and 8 potential points are proposed, which allow for the construction of warehouses, outpatient clinics, hospitals and shelters. The capacity of each of these facilities, the items needed, the healthy and injured people, as well as the probability of injury and the need for treatment in each area, are determined by experts. The results of the implementation of this model is the construction of a hospital in areas 1, 4, 15 and 26; the construction of outpatient centers in areas 3, 11, 13, 24 and 29; the construction of warehouses in the regions 7, 16, 26 and 30, as well as the construction of shelters in Areas 6, 12,25 and 29. Other results from the implementation of the model are given in Table 2. Since the same model that includes the principles of defense is not found, the validation of the answers obtained from the using the experts opinion. So that after the implementation of the model, and achieving results, the results were shared with officials of the Bushehr province. And the logic of the results shows the validity of the model. 
Table 2

Results of model implementation

\begin{tabular}{|c|c|c|c|c|c|c|c|c|c|c|c|c|c|}
\hline & \multicolumn{4}{|c|}{$\delta_{i h}$} & \multicolumn{5}{|c|}{$\psi_{i k}$} & \multicolumn{4}{|c|}{$\varphi_{i s}$} \\
\hline & h1 & h4 & h15 & H26 & k3 & k11 & k13 & k24 & k29 & s6 & s12 & s25 & s29 \\
\hline I1 & & 12 & & & 21 & & & & & & & & 17 \\
\hline I2 & 13 & & & & & & 26 & & & 14 & & & \\
\hline I3 & & 16 & & & 37 & & & & 489 & & 34 & & \\
\hline I4 & & 11 & & & & & & 32 & & & & 24 & \\
\hline I5 & & & 18 & & & 33 & & & & & 29 & & \\
\hline I6 & & 12 & & & & 28 & & & & & & & 23 \\
\hline I7 & & & & 19 & & 0 & & 38 & & 25 & & & \\
\hline I8 & & & & 15 & & 0 & 26 & & & & & 22 & \\
\hline I9 & 10 & & & & & 27 & & & 372 & & & 24 & \\
\hline I10 & 11 & & & & & & & 46 & & & 35 & & \\
\hline I11 & & & 41 & & 53 & & & & & 43 & & & \\
\hline I12 & 15 & & & & & & & 32 & & 25 & & & \\
\hline I13 & & & & 26 & & & & 57 & 671 & & & 39 & \\
\hline I14 & & & 32 & & & 61 & & & & & & & 50 \\
\hline I15 & & & & 25 & & & 48 & & & 33 & & & \\
\hline I16 & & & & 50 & & & 99 & & & & & & 65 \\
\hline I17 & 34 & & & & & 78 & & & 1201 & 45 & & & \\
\hline I18 & 27 & & & & 81 & & & & & & & 74 & \\
\hline I19 & & & & 38 & 47 & & & & & & 53 & & \\
\hline $\mathrm{I} 20$ & & & 24 & & & 40 & & & 667 & & & & 32 \\
\hline I21 & & & 52 & & & & 118 & & & 83 & & & \\
\hline $\mathrm{I} 22$ & 29 & & & & & 56 & & & 815 & & 50 & & \\
\hline I23 & 40 & & & & 53 & & & & & & & 60 & \\
\hline I 24 & & 10 & & & & 16 & & & & & & & \\
\hline $\mathrm{I} 25$ & & 13 & & & & & & 49 & & 47 & & & \\
\hline I26 & & 52 & & & & & & 124 & & & & & 142 \\
\hline I27 & & & & 15 & 17 & & & & 357 & & 22 & & \\
\hline I 28 & & & & 27 & 29 & & & & & & & 34 & \\
\hline I29 & & & 46 & & & 78 & & & & 74 & & & \\
\hline $\mathrm{I} 30$ & & 73 & & & & & 105 & & & & 80 & & \\
\hline
\end{tabular}

\section{Conclusion and suggestions}

One of the most important operations during the crisis is helping the wounded and distributing aid items and transporting people to shelters. The efficiency of this operation is largely due to the decision of managers before the crisis. One of the important decisions before the crisis is location. Different approaches and ideas have been introduced to locate facilities under critical situations in the humanitarian aid chain. In this research, a passive defense approach was used to locate the facility and attempted to find the appropriate location for facilities based on four criteria of population density, user appropriateness, access level and degree of confinement. The results of the running the model showed that aid facilities were constructed in areas with higher population densities, and in that area, more user-friendliness with aid facilities. In addition, these facilities are built in the areas with a higher level of access to roads and at the same time with less confidentiality. In general, GIS is an efficient infrastructure for integrating various data sources, such as spatial information, which can provide a comprehensive database for measuring the efficiency of urban services. 


\section{References}

Akgün, İ., Gümüşbuğa, F., \& Tansel, B. (2015). Risk based facility location by using fault tree analysis in disaster management. Omega, 52, 168-179.

Balcik, B., \& Beamon, B. M. (2008). Facility location in humanitarian relief. International Journal of Logistics, 11(2), 101-121.

Broz, D., Rossit, D., Rossit, D., \& Cavallín, A. (2018). The Argentinian forest sector: opportunities and challenges in supply chain management. Uncertain Supply Chain Management, 6(4), 375-392.

Campbell, A. M., \& Jones, P. C. (2011). Prepositioning supplies in preparation for disasters. European Journal of Operational Research, 209(2), 156-165.

Chen, G., Daskin, M. S., Shen, Z. J. M., \& Uryasev, S. (2006). The $\alpha$-reliable mean-excess regret model for stochastic facility location modeling. Naval Research Logistics (NRL), 53(7), 617-626.

Cozzolino, A. (2012). Humanitarian logistics: cross-sector cooperation in disaster relief management. Springer Science \& Business Media.

Dessouky, M., Ordonez, F., Jia, H., \& Shen, Z. (2006). Rapid distribution of medical supplies. In Patient Flow: Reducing Delay in Healthcare Delivery (pp. 309-338). Springer US.

Esmaeili, V., \& Barzinpour, F. (2014). Integrated decision making model for urban disaster management: A multi-objective genetic algorithm approach. International Journal of Industrial Engineering Computations, 5(1), 55-70.

Ghaffari, A. (1988). Fundamentals design of Educational Spaces. Tehran. Organization of the renovation and equipping of schools in the country.1998.

Hall, R. (Ed.). (2012). Handbook of transportation science(Vol. 23). Springer Science \& Business Media.

Jia, H., Ordóñez, F., \& Dessouky, M. (2007). A modeling framework for facility location of medical services for large-scale emergencies. IIE transactions, 39(1), 41-55.

Kawasaki, A., Berman, M. L., \& Guan, W. (2013). The growing role of web-based geospatial technology in disaster response and support. Disasters, 37(2), 201-221.

Hooshiyar H. (2010). Locating of therapeutic uses using the AHP method (Case study: Mahabad city). Geographical Space Journal, 131150.

Hosseini, A, Modiri, M., \& Hooshang, M. (2004). Assessment of the distribution and access of citizens to emergency services for human incidents with passive defense approach (Case study of 117 areas of Tehran). Geographical Space Journal, 2, 173192.

Jafar Nejad, A., Hashemi, H., \& Talaei, H. (2013). New Approaches to Supply Chain Management, Tehran.

Jalali Farahani, Gh. (2011). Territorial action from the perspective of passive defense. Tehran. 2011.

Kunz, N., \& Reiner, G. (2012). A meta-analysis of humanitarian logistics research. Journal of Humanitarian Logistics and Supply Chain Management, 2(2), 116-147.

Naji-Azimi, Z., Renaud, J., Ruiz, A., \& Salari, M. (2012). A covering tour approach to the location of satellite distribution centers to supply humanitarian aid. European Journal of Operational Research, 222(3), 596-605.

Nidhi, M., \& Pillai, V. (2017). Development of a parametric matrix based on GSCM literature. Accounting, 3(1), 53-80.

Oloruntoba, R., \& Gray, R. (2006). Humanitarian aid: an agile supply chain?. Supply Chain Management: an international journal, 11(2), 115-120.

Rawls, C. G., \& Turnquist, M. A. (2011). Pre-positioning planning for emergency response with service quality constraints. OR spectrum, 33(3), 481-498.

Rawls, C. G., \& Turnquist, M. A. (2012). Pre-positioning and dynamic delivery planning for shortterm response following a natural disaster. Socio-Economic Planning Sciences, 46(1), 46-54.

Schulz, S. F., \& Blecken, A. (2010). Horizontal cooperation in disaster relief logistics: benefits and impediments. International Journal of Physical Distribution \& Logistics Management, 40(8/9), 636-656. 
Van Wassenhove, L. N. (2006). Humanitarian aid logistics: supply chain management in high gear. Journal of the Operational Research Society, 57(5), 475-489.

Yamane, K. (2018). Downward-sloping linear supply function equilibrium in duopoly. Journal of Project Management, 3(3), 151-156.

Zhang, Y., Liu, W., Lou, W., \& Fang, Y. (2006). Location-based compromise-tolerant security mechanisms for wireless sensor networks. IEEE Journal on Selected Areas in Communications, 24(2), 247-260.

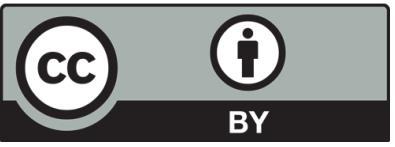

(C) 2018 by the authors; licensee Growing Science, Canada. This is an open access article distributed under the terms and conditions of the Creative Commons Attribution (CC-BY) license (http://creativecommons.org/licenses/by/4.0/). 